Supporting information for

Aspheric Solute Ions Modulate Gold Nanoparticle Interactions in an Aqueous Solution: An Optimal Way to Reversibly Concentrate Functionalized Nanoparticles

Oscar D Villarreal, Liao Y Chen, Robert L Whetten and Borries Demeler

\title{
Building procedure
}

The NP was built up from the structure of the $A u_{144} S_{60}$ core taken from Refs.[1-3]. The outer $\mathrm{Au}_{90} \mathrm{~S}_{60}$ were bound covalently as shown in Fig. S1, left. 60 copies of the 4-mercaptobenzoate thiolate group $\mathrm{SPhCOO}^{-}$were then made without the sulfur atom, so as to bind them with the sulfurs at the NP surface, as shown in Fig. S1, middle. The potential energy of the result was then lowered in vacuum while keeping the $A u_{144} S_{60}$ core fixed. The NP was then paired up with a copy of itself placed at a center-to-center distance of $4 \mathrm{~nm}$ along the $\mathrm{z}$ axis. The pair was solvated in a water box of size either 10x10x20 nm or 10x10x15 nm, neutralized with $\mathrm{Na}^{+} / \mathrm{K}^{+} / \mathrm{TMA}^{+} / \mathrm{TRS} S^{+}$counter-ions and additionally salinated with 0 / 50 / $100 \mathrm{mM}$ of $\mathrm{NaOH} / \mathrm{KOH} / \mathrm{TMAOH} / \mathrm{TRSCl}$ (see Fig. S1, right). The result was then minimized again and equilibrated at constant temperature (298 K) and pressure (1 bar) for $20 \mathrm{~ns}$ while keeping the innermost $\mathrm{Au}_{12}$ atoms of both cores fixed.

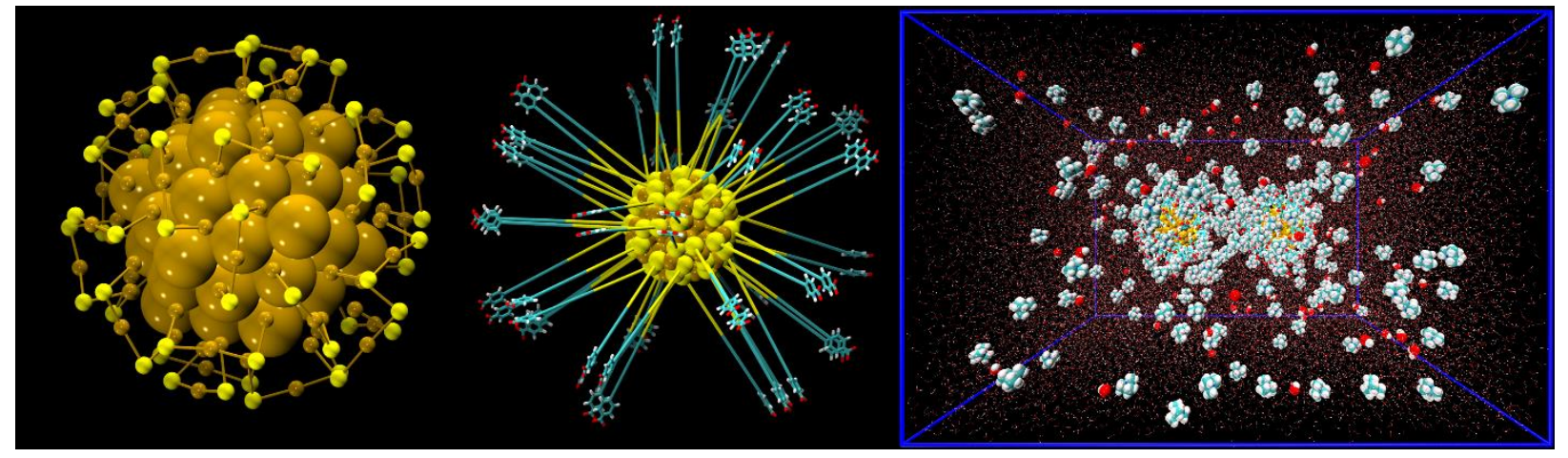

Fig. S1. Left: The $\mathrm{Au}_{144} \mathrm{~S}_{60}$ core with the inner $\mathrm{Au}_{54}$ represented as spheres with van der Waals radii and the outer $\mathrm{Au}_{90} \mathrm{~S}_{60}$ as ball-and-stick. Middle: the 60 ligands bound to the sulfur atoms prior to energy minimization in vacuum. Right: the 10x10x15nm water box containing two $\mathrm{Au}_{144}\left(\mathrm{SPhCOO}^{-}\right)_{60} \mathrm{NPs}$, neutralized with $120 \mathrm{TMA}^{+}$ions and salinated by $100 \mathrm{mM}$ TMAOH, for a total of 138637 atoms. 


\section{Calculation of the standard binding free energy}

Following the methodology of Ref.[4], after equilibrating the NP pairs for $20 \mathrm{~ns}$, the potential of mean force (PMF) was computed as they were dragged towards each other until the interaction became strongly repulsive. The inner $12 \mathrm{Au}$ atoms at the core of each NP were pulled simultaneously both towards and away from each other through NAMD[5], as adapted for steered molecular dynamics (SMD) at a speed of $1 \mathrm{~nm} / \mathrm{ns}$ at intervals of 2 each. At the end of each interval the system was allowed to reach equilibrium for $1 \mathrm{~ns}$. Within each interval, eight paths were computed: four in the forward direction and four in the reverse direction, as shown in Fig. S2. From each of these paths the mechanical work employed to drag the NPs was measured.

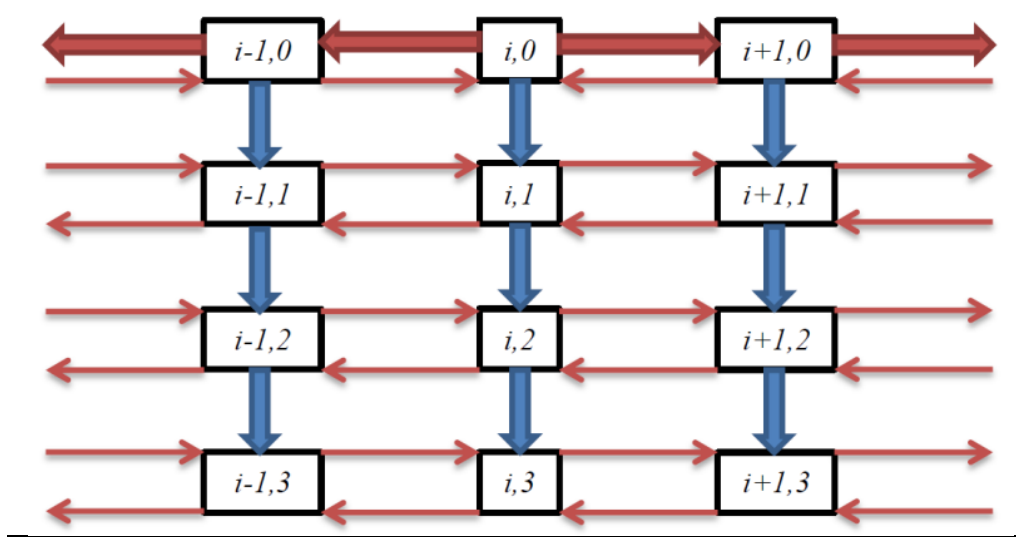

Fig. S2. Starting from state 'I,0' (interval I, path 0 ), the broad red arrows represent $0.1 \mathrm{~ns}$ of dragging the center of mass of each NP forward / backwards at a speed of $1 \mathrm{~nm} / \mathrm{ns}$, followed by $1 \mathrm{~ns}$ of equilibration to generate the ' $\mathrm{i} \pm 1, \mathrm{j}$ ' state; the blue arrows represent 10 ps of equilibration; and the narrow red arrows represent the same dragging as the broad red arrows, but without equilibrating the state at the end. A work profile is obtained from each red arrow.

The PMF[6-11] as a function of the separation between the 12 Au inner cores was then computed through the Brownian-dynamics fluctuation-dissipation theorem:[12]

$$
P M F(z+\Delta z)-P M F(z)=-k_{B} T \ln \left(\frac{\left\langle\exp \left[-W_{z \rightarrow z+\Delta z} / 2 k_{B} T\right]\right\rangle}{\left\langle\exp \left[-W_{z+\Delta z \rightarrow z} / 2 k_{B} T\right]\right\rangle}\right)
$$

where $k_{B}$ is the Boltzmann constant, $\mathrm{T}$ is the absolute temperature, $W_{z \rightarrow z+\Delta z}$ is the work done on the system as the NPs are pulled towards each other by $\Delta z=0.2 \mathrm{~nm}$ and $W_{z+\Delta z} \rightarrow z$ 
is the work done as they are pulled away from each other by the same amount. The dissociation constant was computed following the standard literature $[13,14]$ as follows:

$$
k_{D}=c_{0} / \int_{0}^{r^{* *}} d r 4 \pi r^{2} \exp \left[-P M F(r) / k_{B} T\right] c_{0}
$$

where $c_{0}=1 M=6.023 \times 10^{-1} \mathrm{~nm}^{-3}$ is the standard concentration and $r^{*}$ is the cut-off separation beyond which the AuNPs are considered in the dissociated state. The standard binding energy is then:

$$
\Delta G=k_{B} T \ln \left[k_{D} / c_{0}\right]=-k_{B} T \ln \left[c_{0} \int_{0}^{r^{*}} d r 4 \pi r^{2} \exp \left[-P M F(\mathrm{r}) / k_{B} T\right]\right]
$$

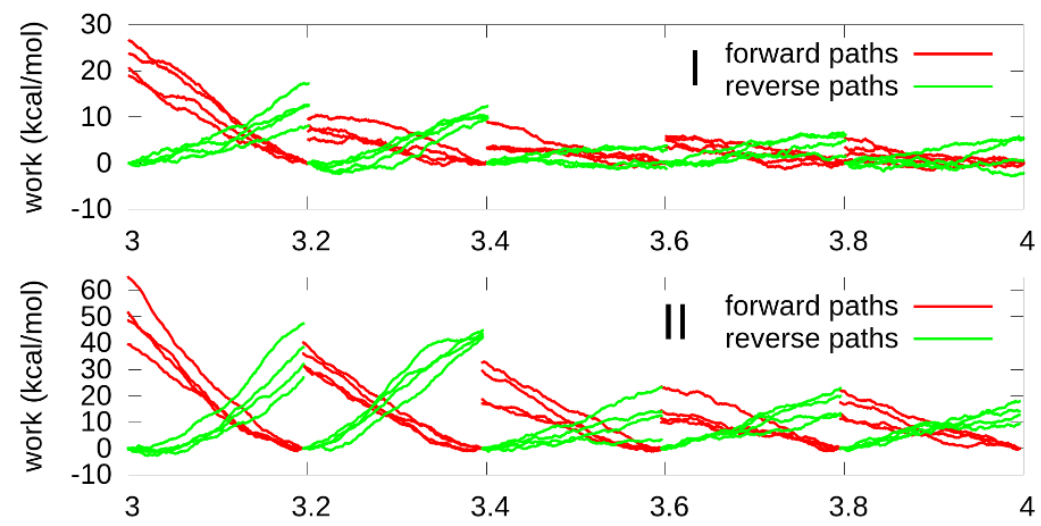

Fig. S3. The work along forward and reverse paths of pulling two NPs together as a function of the separation $\mathrm{r}(\mathrm{nm})$ between the centers of mass of the Au 12 inner cores.
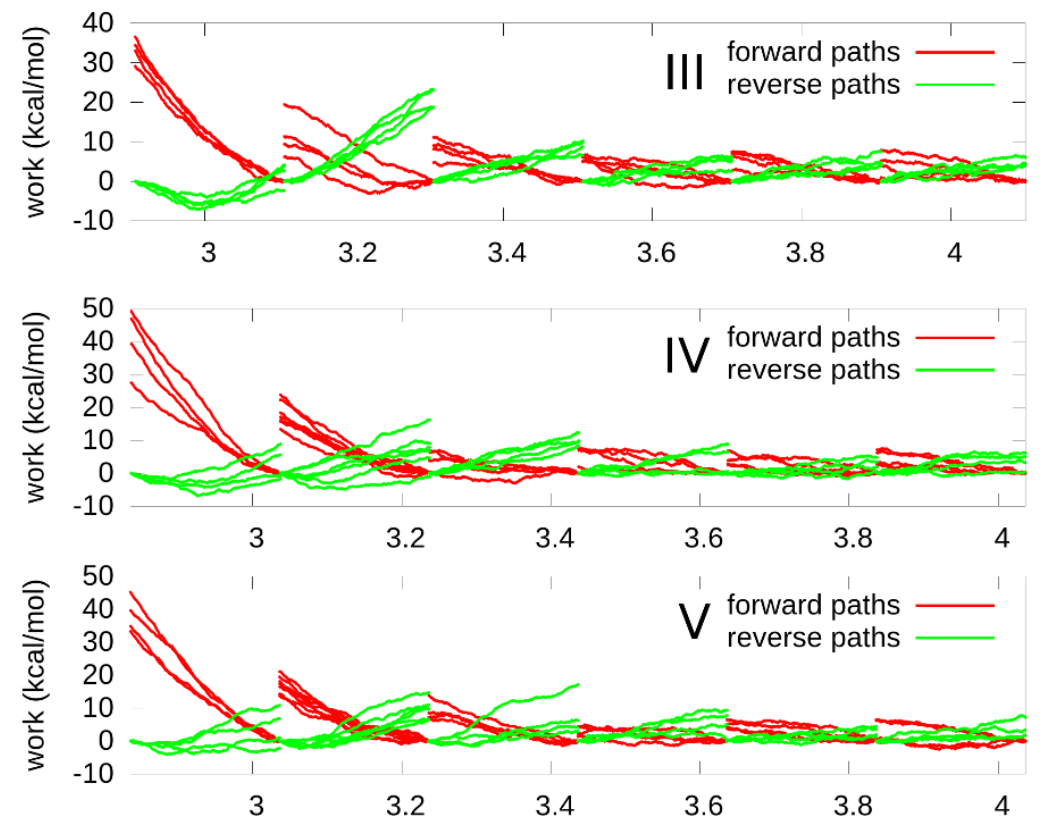

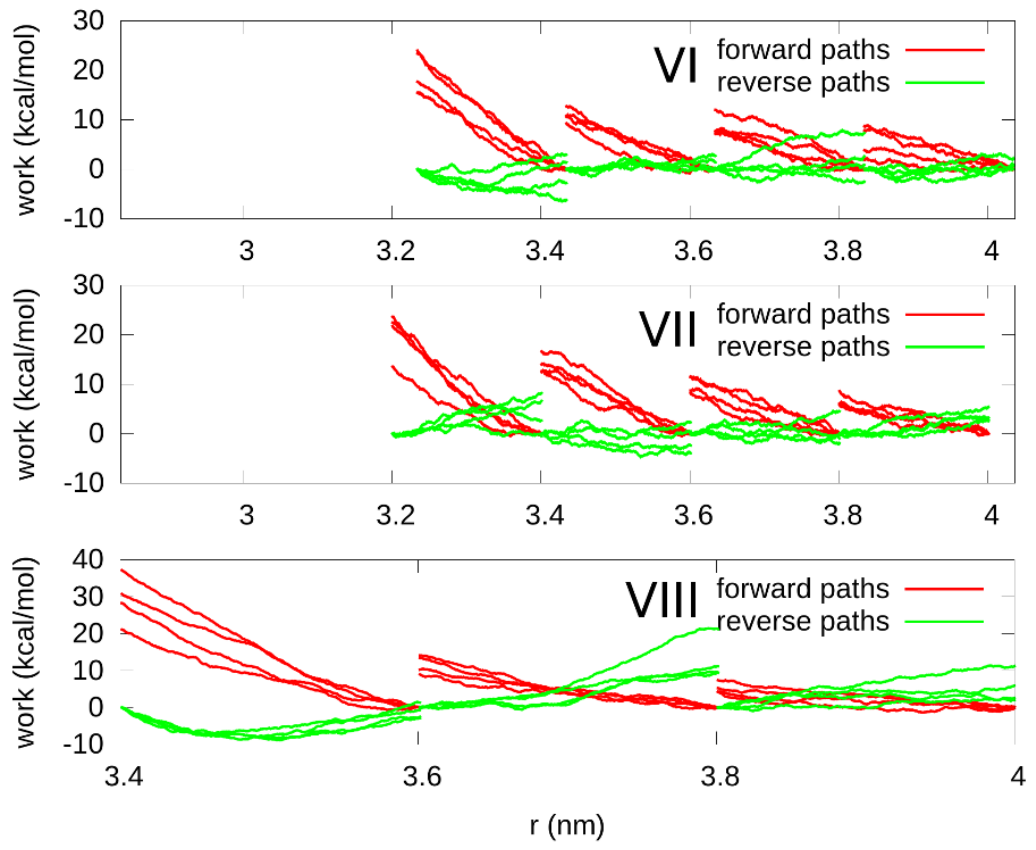

\section{Effect of the electrolyte's size on its interactions}

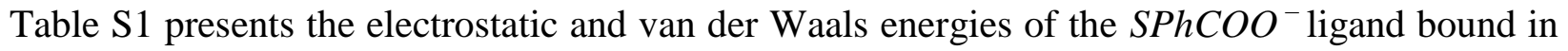
vacuum to one cation of each type, in their configuration of minimum energy. The center-of-mass $(\mathrm{COM})$ of $\mathrm{TMA}^{+}$was $1.5 \AA$ farther from the $\mathrm{COO}^{-}$terminal group than the $\mathrm{COM}$ of $\mathrm{Na}^{+}$, consequently decreasing significantly the Coulombic interaction. Also shown are the potentials of mean force (PMFs) as the cations were pulled from the ligands inside of a water box. The results indicate that $\mathrm{Na}^{+}$binds the strongest to the ligand, and $\mathrm{TMA}^{+}$the weakest. 
Table S1. The electrostatic and van der Waals energies of the SPhCOO- thiolate group bound in vacuum with the corresponding counterion at the state of minimum energy, as well as the separation between the carbon of the terminal group and the center-of-mass of the counterion.

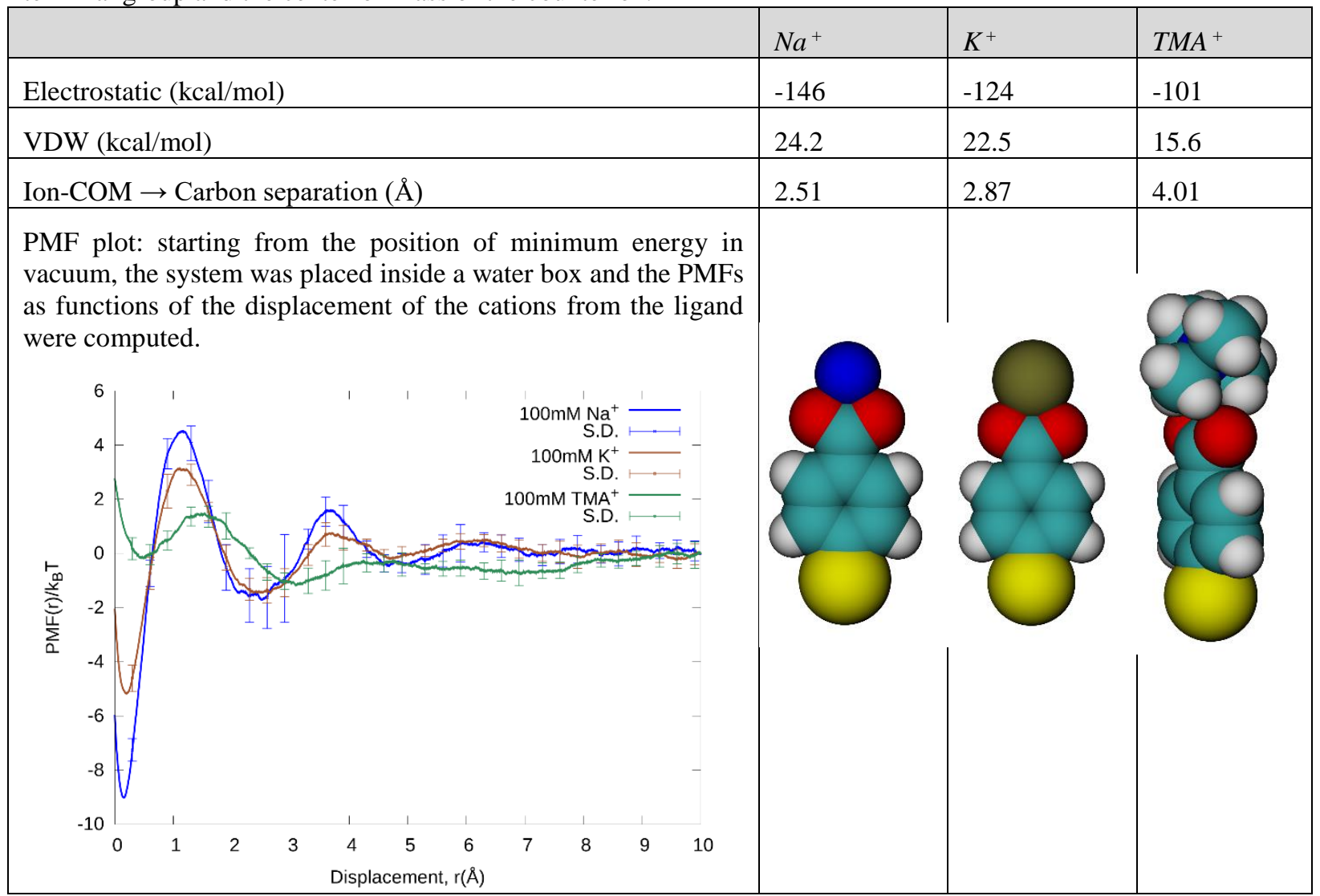

A result of the difference in van der Waals radii of the counterions $\left(0.136 \mathrm{~nm}\right.$ for $\mathrm{Na}^{+}, 0.176 \mathrm{~nm}$ for $\mathrm{K}^{+}$and $\approx 0.23 \mathrm{~nm}$ for $T M A^{+}$), shown in Fig. $\mathrm{S} 4$, is that the peak of the first hydration shell is highest for $\mathrm{Na}^{+}$and lowest for $\mathrm{TMA}^{+}$. For these equally charged ions, the larger the size, the lower the charge density and thus the weaker the interactions with water became. Additional 20ns simulations were performed on isolated nanoparticles in a 10x10x10nm water box, neutralized with 120 counterions and further ionized with a $150 \mathrm{mM}$ concentration of electrolyte. The resulting distribution of counterions, also shown in Fig. S4, yields a highest peak for sodium, which is due to the stronger affinity it has to the NP's surface. Yet as pointed out in Ref. [15], the repulsion between pairs of hydrated $\mathrm{Na}^{+}$ions is stronger than between hydrated $\mathrm{K}^{+}$ions, which would hinder their close packing. We have here thus two opposing interactions: the ion-ion repulsion 
$\left(\mathrm{Na}^{+}>\mathrm{K}^{+}\right)$which hinders close packing, and the ion-ligand attraction $\left(\mathrm{Na}^{+}>\mathrm{K}^{+}\right)$which favors it, and Fig. S4 results from the balance between these forces.
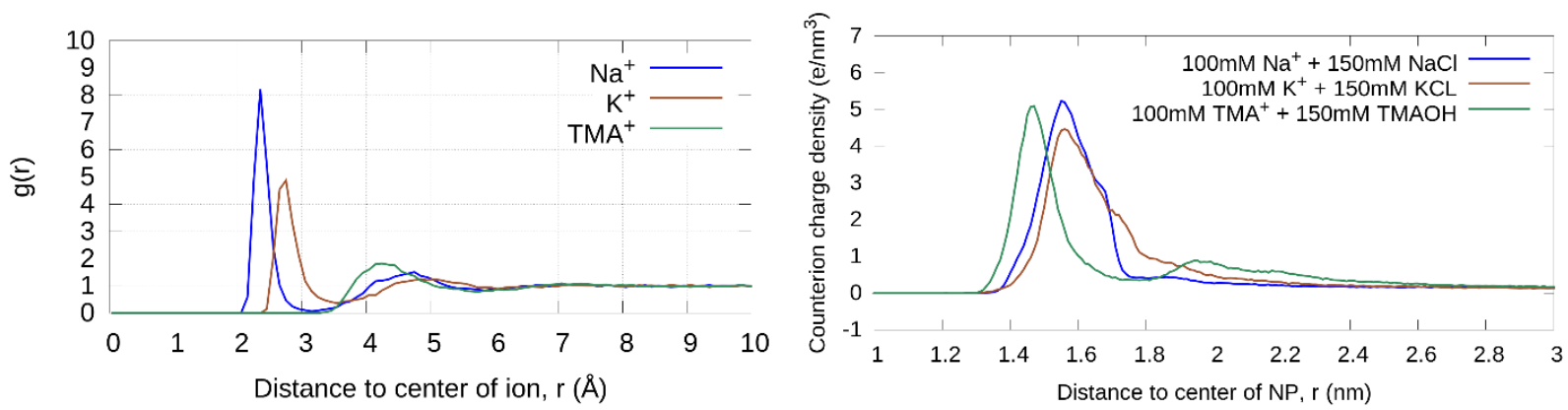

Fig. S4. Left: The radial pair distribution function $\mathrm{g}(\mathrm{r})$ for each type of counterion with water, obtained from $1 \mathrm{~ns}$ simulations in a $3 \times 3 \times 3 \mathrm{~nm}$ water box. Right: the distribution of counterions as a function of radial distance to the center of the NP, obtained from $20 \mathrm{~ns}$ simulations in a $10 \times 10 \times 10 \mathrm{~nm}$ water box.

The counterion distributions around the AuNPs were fitted to the Debye-Hückel description around a charged NP: $A r^{-1} e^{-} B r+C$ where $B^{-1}$ is the Debye length[2], and the results are shown in table $\mathrm{S} 2$.

Table S2. The counterion density distributions were fitted to the Debye-Hückel description around a charged nanoparticle. The potassium distribution approached the Debye-Hückel description the most. The distribution of sodium became sharply horizontal at $\approx 1: 7 \mathrm{~nm}$ since it was more strongly bound to the nanoparticle, whereas the distribution of TMA+ presented a second peak at $\approx 1: 9 \mathrm{~nm}$.

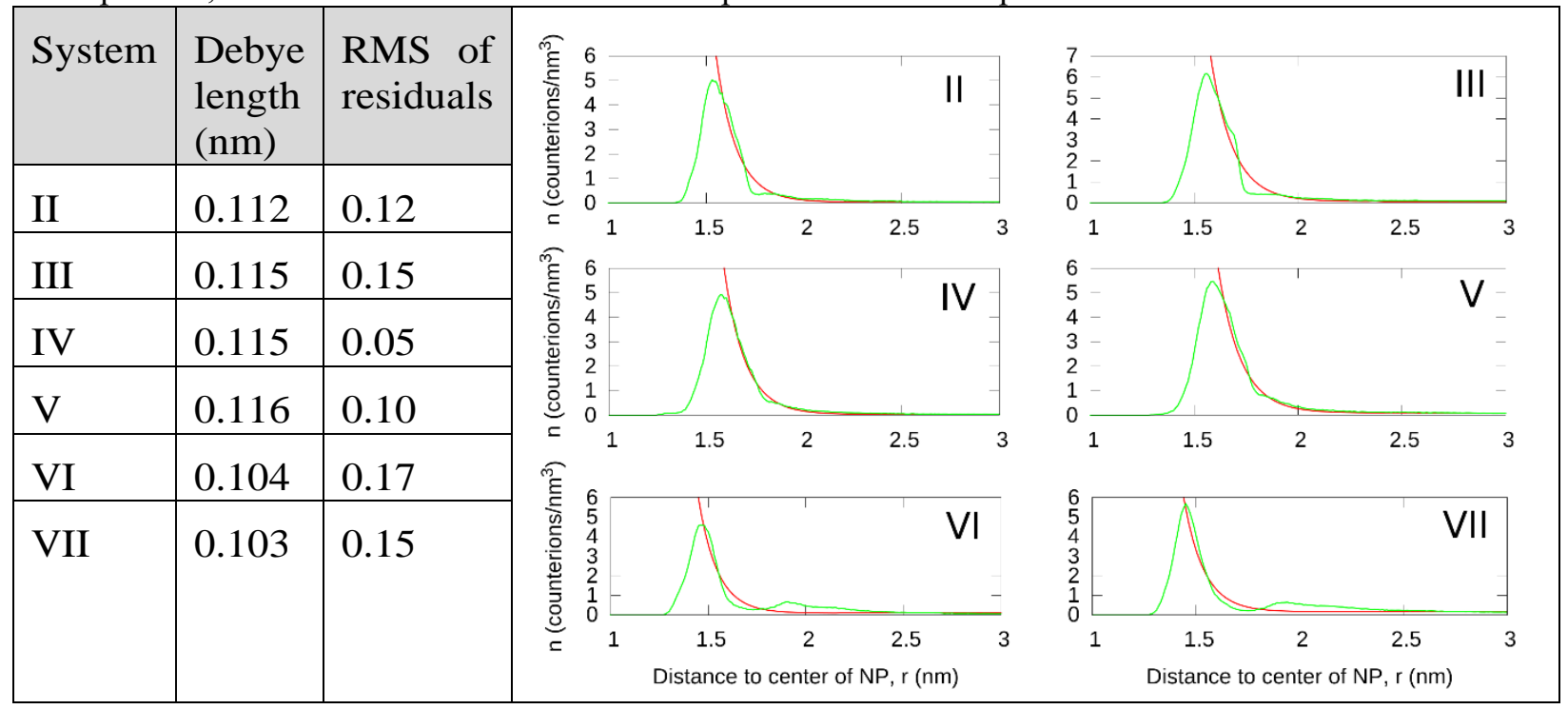

The main origin of the attraction between the NPs was observed to be the electrostatic interaction between their negative terminal groups and the positive cation cloud lying at the 
region in-between them. As seen in Fig. S5, the direct van der Waals attraction did not appear to play a large role.

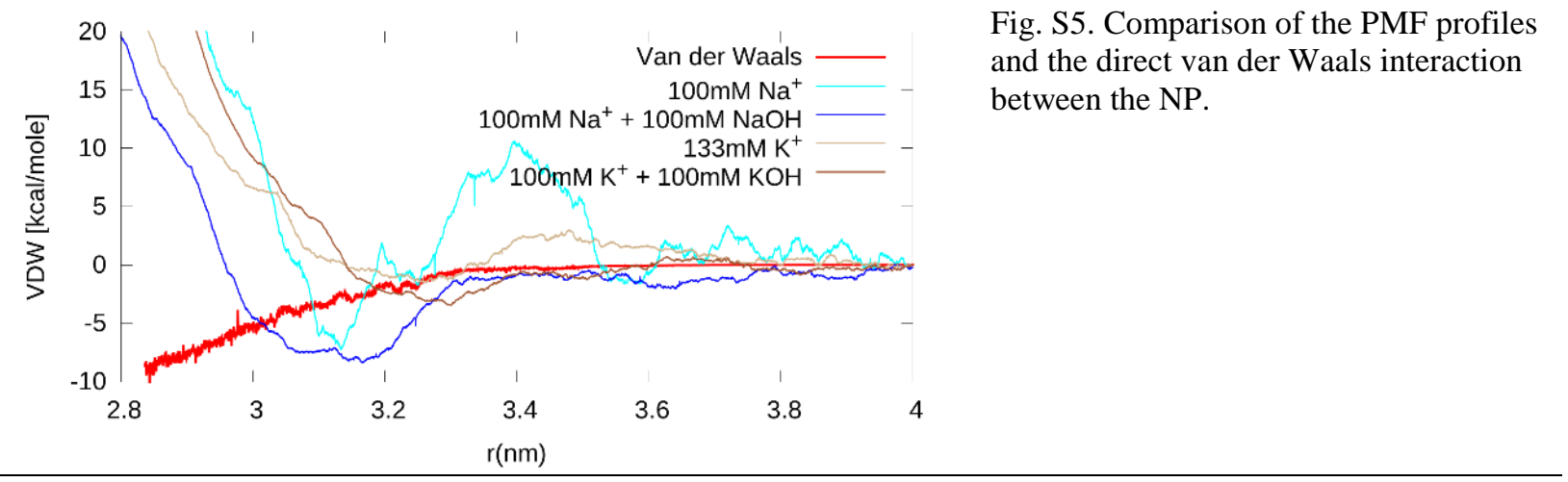

In order to observe the difference in hydrophilic behavior between $\mathrm{Na}^{+}$and $\mathrm{TMA}^{+}$we computed their hydration energy following ref.[16] in the following way: we placed each ion in a vacuum at a distance of $1 \mathrm{~nm}$ from a water box of size $6 \times 6 \times 6 \mathrm{~nm}$ and dragged them into the box while measuring the potential of mean force, as shown in Fig. S6. $\mathrm{Na}^{+}$changed by $-91.2 \mathrm{kcal} / \mathrm{mol}$ and $\mathrm{TMA}^{+}$changed by $-41.4 \mathrm{kcal} / \mathrm{mol}$. To this must be added the freeenergy difference corresponding to the Coulomb interaction as the ion approaches from infinity:

$$
G_{0}-G_{\infty}=-\frac{q^{2}}{16 \pi \varepsilon_{0}\left|z_{0}\right|}\left(1-\frac{2 \varepsilon_{0}}{\varepsilon_{0}+\varepsilon}\right)
$$

where $\varepsilon_{0}$ is the permittivity of vacuum, $q=e$ is the ionic charge, $z_{0}=-1 \mathrm{~nm}$ is the initial position of the ions and $\varepsilon=80 \varepsilon_{0}$ is water's permittivity, thus:

$$
G_{0}-G_{\infty}=-\frac{\left(1.602 \times 10^{-19} \mathrm{C}\right)^{2}}{16 \pi \times 8.854 \times 10^{-12} \mathrm{~F} / \mathrm{m} \times 1 \times 10^{-9} \mathrm{~m}} \times\left(1-\frac{2}{81}\right) \times \frac{6.02 \times 10^{23} \mathrm{~mol}^{-1}}{4184 \mathrm{~J} / \mathrm{kcal}}=-8.1 \mathrm{kcal} / \mathrm{mol}
$$


The computed hydration energies are then $\sim 100 \mathrm{kcal} / \mathrm{mol}$ for $\mathrm{Na}^{+}$and $\sim 50 \mathrm{kcal} / \mathrm{mol}$ for $\mathrm{TMA}^{+}$, as expected when considering the fact that $\mathrm{TMA}^{+}$'s surface is covered by hydrocarbons.

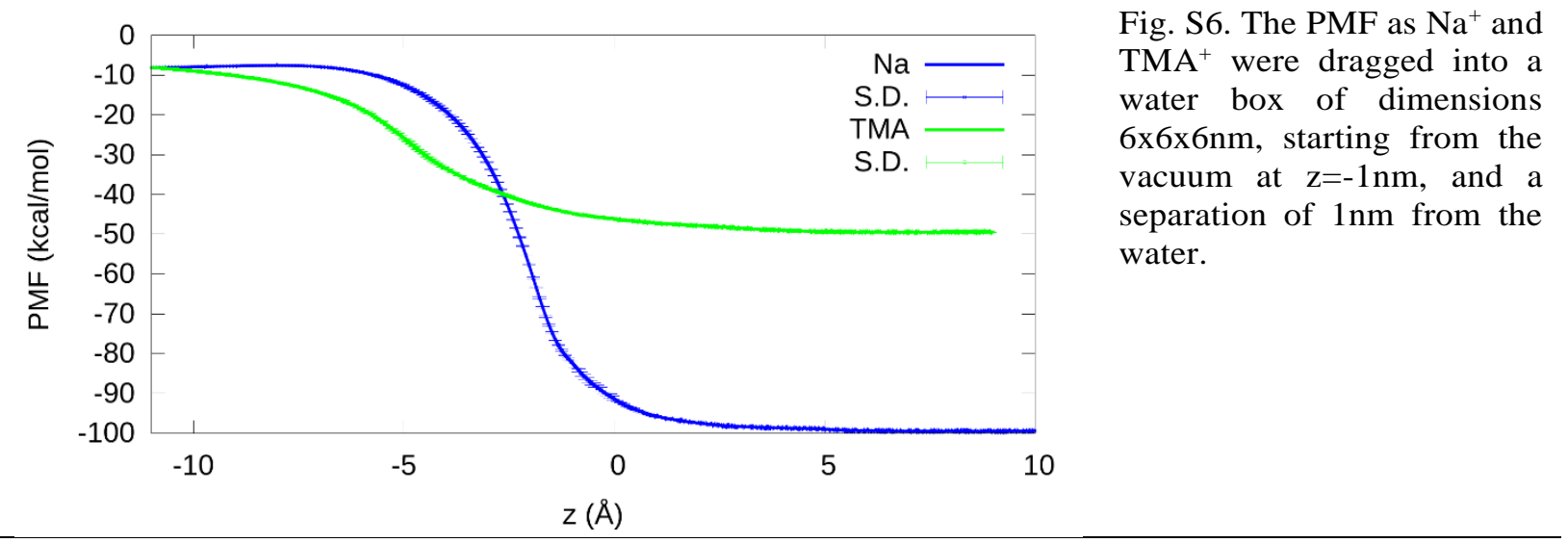

\section{Effect of the electrolyte's size on the NP-NP interactions}

All equilibrium properties of the systems containing pairs of NPs were measured from the last half of the 20ns simulation done under equilibrium conditions. The root mean squared deviation (RMSD) of the $\mathrm{COO}^{-}$terminal groups was $\approx 1 \AA$ in all cases, as shown in Fig. S7, which denotes the high rigidity of the $\mathrm{SPhCOO}{ }^{-}$ligand. Such rigidity facilitates the binding of the counterions to the terminal groups. The RMSD of the inner $A u_{114}$ core was $\approx 0.02 \mathrm{~nm}$ and of the outer $A u_{30} S_{60}$ shell was $\approx 0.03 \mathrm{~nm}$, thus verifying the stability of the core.

In Fig. S7 we show the radial concentration of the terminal groups as function of the distance to the center of the NPs, as well as the pair correlation function $g(r)$ of water with the surface of the NPs, the RMSD of the terminal groups and the electrostatic potential along the centers of mass of both NPs. 
The peaks in the pair correlation with water are higher for a concentration of $0 \mathrm{M}$ in every case, since the fewer amount of counterions interfered less with the water structure generated by the NP around itself. Sodium allowed higher peaks than potassium, as expected from its more strongly bound hydration shell (it was able to bring more water to the surface). The highest peak corresponds to using $120 \mathrm{TMA}^{+}$, since this case had the lowest counterion concentration at the surface. On the other hand, the lowest peak corresponds to adding $100 \mathrm{mM}$ of TMAOH to the neutralized system, likely because $T M A^{+}$has the weakest-bound hydration shell and thus brings less water to the surface.
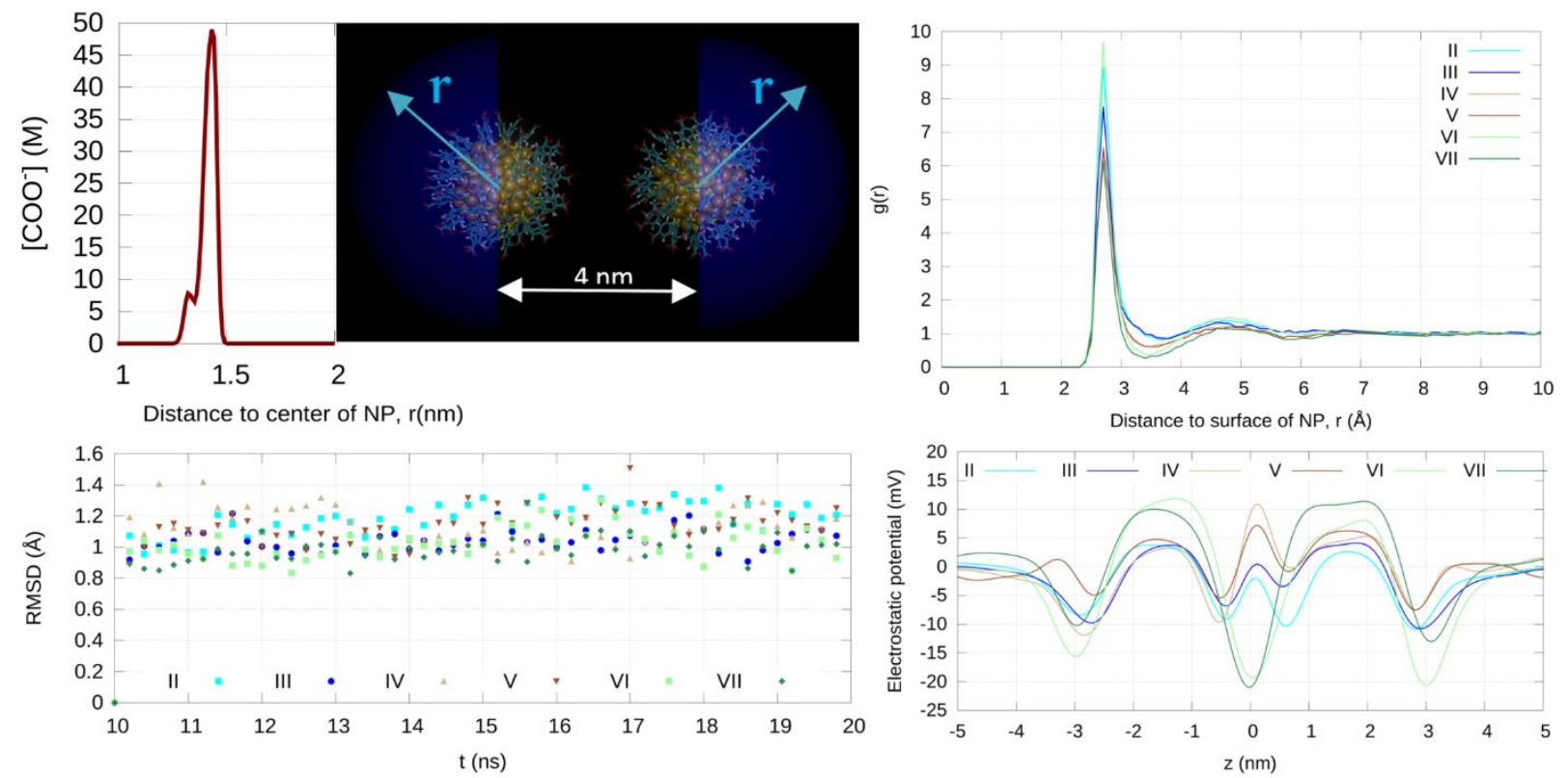

Fig. S7. Top left: The radial concentrations of the terminal groups as functions of the distance to the centers of the NPs. Top right: The water-NP pair correlations with respect to the NP's surface (consisting of all ligand atoms as well as the outermost $\mathrm{S}-\mathrm{Au}-\mathrm{S}$ layer, i.e. the horizontal axis is not a radial distance). These measurements were made in the regions colored with blue in the snapshot. Bottom left: the RMSD values of the $60 \mathrm{COO}^{-}$terminal groups. Bottom right: the electrostatic potential along the $\mathrm{z}$ axis, passing through the COM of both NPs, computed through the PMEpot[44] plugin of VMD, with an Ewald factor of 0:25A. The center-to-center separation corresponded to the location of the minimum in the PMF $(3.1 \mathrm{~nm}$ for $\mathrm{NaOH}$, and $3.2 \mathrm{~nm}$ for $\mathrm{KOH}$; since TMAOH had no minimum, the separation was also $3.2 \mathrm{~nm}$ in order to compare with $\mathrm{KOH}$ ). 


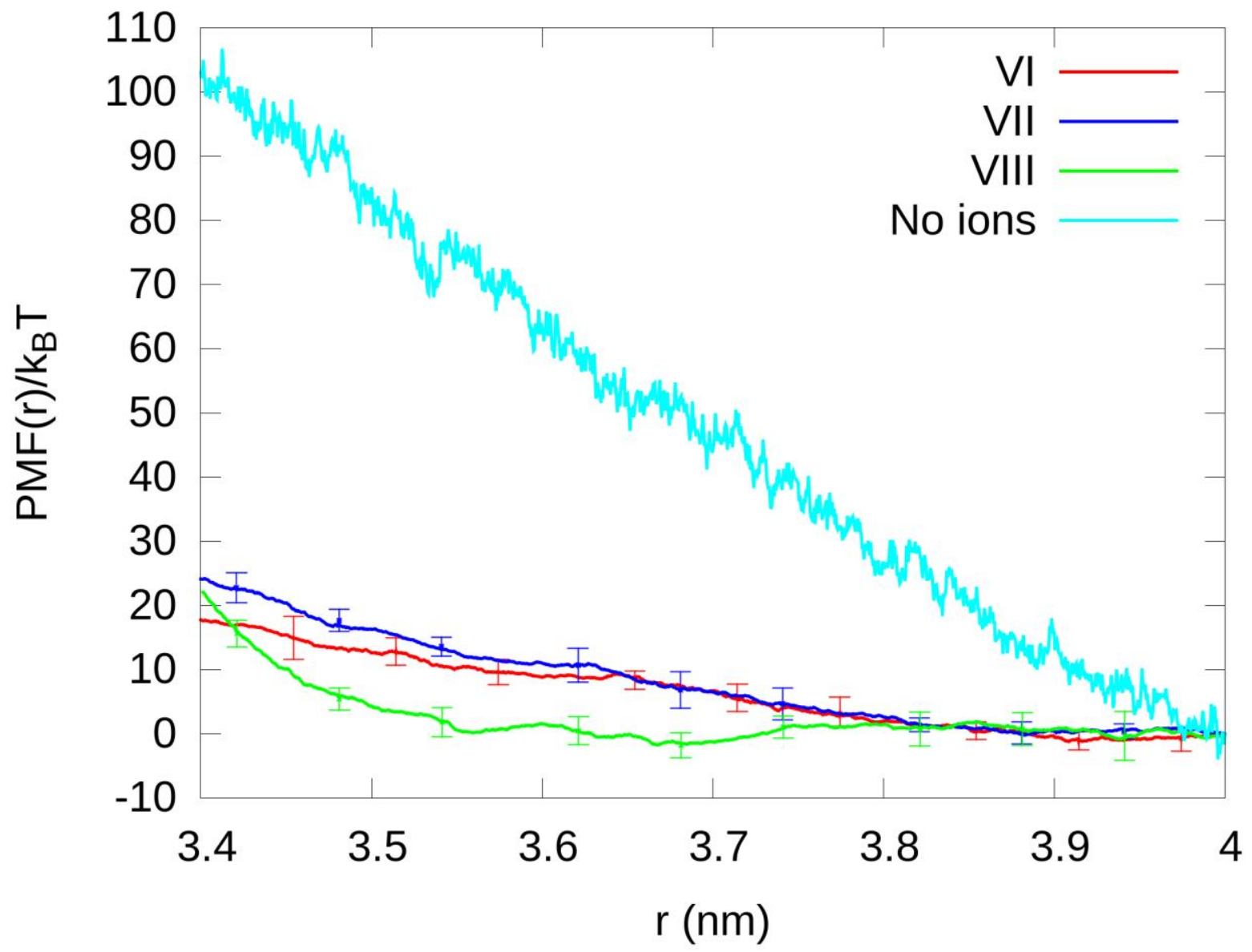

Fig. S8. PMF curves of System VI to VIII vs. that of a system of pure water without any counterions.

\section{References}

1. Bahena, D., et al., STEM Electron Diffraction and High Resolution Images Used in the Determination of the Crystal Structure of Au(SR) Cluster. J Phys Chem Lett, 2013. 4(6): p. 975-981.

2. Lopez-Acevedo, O., et al., Structure and Bonding in the Ubiquitous Icosahedral Metallic Gold Cluster Au144(SR)60. The Journal of Physical Chemistry C, 2009. 113(13): p. 5035-5038.

3. Tlahuice-Flores, A., et al., Structure and bonding of the gold-subhalide cluster IAu144Cl60[z]. Phys Chem Chem Phys, 2013. 15(44): p. 19191-5.

4. Chen, L.Y., Glycerol modulates water permeation through Escherichia coli aquaglyceroporin GlpF. Biochim Biophys Acta, 2013. 1828(8): p. 1786-93. 
5. $\quad$ Phillips, J.C., et al., Scalable molecular dynamics with NAMD. J Comput Chem, 2005. 26(16): p. 1781-802.

6. $\quad$ Allen, T.W., O.S. Andersen, and B. Roux, Molecular dynamics - potential of mean force calculations as a tool for understanding ion permeation and selectivity in narrow channels. Biophys Chem, 2006. 124(3): p. 251-67.

7. Chandler, D., Statistical mechanics of isomerization dynamics in liquids and the transition state approximation. The Journal of Chemical Physics, 1978. 68(6): p. 29592970.

8. Kirkwood, J.G., Statistical mechanics of fluid mixtures. Journal of Chemical Physics, 1935. 3(5): p. 300-313.

9. Pratt, L.R., G. Hummer, and A.E. Garcia, Ion-Pair Potentials-of-Mean-Force in Water. Biophysical Chemistry, 1994. 51(2-3): p. 147-165.

10. Roux, B., The Calculation of the Potential of Mean Force Using Computer-Simulations. Computer Physics Communications, 1995. 91(1-3): p. 275-282.

11. Wu, J.Z., Density functional theory for chemical engineering: From capillarity to soft materials. Aiche Journal, 2006. 52(3): p. 1169-1193.

12. Chen, L.Y., Nonequilibrium fluctuation-dissipation theorem of Brownian dynamics. J Chem Phys, 2008. 129(14): p. 144113.

13. Deng, Y. and B. Roux, Computations of standard binding free energies with molecular dynamics simulations. J Phys Chem B, 2009. 113(8): p. 2234-46.

14. Jorgensen, W.L., Interactions between Amides in Solution and the Thermodynamics of Weak Binding. Journal of the American Chemical Society, 1989. 111(10): p. 3770-3771.

15. Pashley, R.M., $\backslash L L O \backslash$ and hydration forces between mica surfaces in $\mathrm{Li+}, \mathrm{Na}+\mathrm{K}+$, and Cs+electrolyte solutions: A correlation of double-layer and hydration forces with surface cation exchange properties Journal of Colloid and Interface Science 1981. 83(2): p. 531 - 546.

16. Chen, L.Y., Exploring the free-energy landscapes of biological systems with steered molecular dynamics. Physical Chemistry Chemical Physics, 2011. 13(13): p. 6176-6183. 